SCIENTIFIC REPORT

\title{
Activation of nuclear factor-kappa B in the conjunctiva with the epithelial scraping of the mouse cornea and human epidemic keratoconjunctivitis
}

\author{
S Kase, K Aoki, T Harada, C Harada, K Ohgami, K Shiratori, S Nishi, S Ohno, K Yoshida
}

Br J Ophthalmol 2004;88:947-949. doi: 10.1136/bjo.2003.024646

\begin{abstract}
Aim: To examine the expression of $\mathrm{p} 65$, one of nuclear factor-kappa B (NF- $\kappa B$ ), in the conjunctival epithelium of the C57Bl6 mouse and a patient with epidemic keratoconjunctivitis (EKC).

Methods: Normal and epithelial scraped cornea obtained 6 hours after the injury were processed for paraffin section. Samples of a normal and an EKC conjunctival epithelium were obtained using impression cytology. Both samples were analysed by immunocytochemistry using anti-p65 antibody. Results: Immunocytochemistry with the anti-NF- $\mathrm{kB}$ p65 antibody revealed that p65 was localised in the cytoplasm of the conjunctival epithelium in the C57Bl6 mouse without the treatment. Six hours after the scraping of the cornea, p65 protein was expressed in the nuclei of the conjunctival epithelium. p65 was localised in the cytoplasm of the conjunctival epithelium in the human normal eye. p65 protein was expressed in the nuclei of the conjunctival epithelial cells in the EKC patient.

Conclusion: These findings suggest that NF- $\kappa B$ was activated in the conjunctiva in the epithelial scraping of the mouse cornea and in human EKC.
\end{abstract}

$\mathrm{E}$ pidemic keratoconjunctivitis (EKC) is a relatively severe virus infection involving both the conjunctiva and the cornea. ${ }^{1}$ A limited number of adenoviruses (adenovirus (Ad) type 8, Ad19, and Ad37) are frequently isolated from patients suffering from EKC. ${ }^{34}$

In cases of EKC and corneal erosion, strong inflammation in the conjunctiva is noted. Previous reports demonstrated that corneal ulcers healed after the excision of conjunctival epithelium, ${ }^{56}$ indicating that conjunctiva have a critical role in corneal inflammation. Tumour necrosis factor (TNF) $\alpha$ and interleukin (IL)-1 $\beta$ were induced during corneal inflammation, ${ }^{78}$ suggesting that regulation of synthesis of cytokines affect inflammation in the eye.

Nuclear factor $(\mathrm{NF})-\kappa \mathrm{B}$ protein is one of the major transcription factors. ${ }^{9}{ }^{10}$ The activation of NF- $\mathrm{\kappa B}$ leads to synthesis of proinflammatory cytokines including TNF- $\alpha$ and IL- $1 \beta$, which mediate inflammatory and immune responses. ${ }^{11}$ $\mathrm{NF}-\kappa \mathrm{B}$ protein is regulated by interaction with $\mathrm{I}-\kappa \mathrm{B}$ inhibitors $(\mathrm{I}-\kappa \mathrm{Bs}) .{ }^{910}$ In non-stimulated cells, NF- $\mathrm{KB}$ proteins are retained in the cytoplasm because I-кBs mask their nuclear localisation sequence. Exposure to proinflammatory stimuli results in rapid phosphorylation, ubiquitination, and degra-

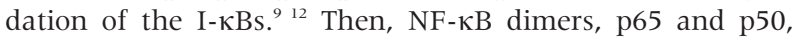
translocate to the nucleus and regulate target gene transcription. It was reported that proinflammatory cytokines including TNF- $\alpha$ and IL- $1 \beta$ activated NF- $\kappa B .{ }^{9}$ Thus, products of the genes that are regulated by NF- $\kappa \mathrm{B}$ also cause the activation of $\mathrm{NF}-\kappa \mathrm{B} .{ }^{11}$ This type of positive regulatory loop may amplify and perpetuate local inflammatory responses.

It was reported that the E3 promoter of human adenovirus had binding sites for NF- $\mathrm{KB}$, which appeared to be a cassette of genes that functions to counteract the host's antiviral defences. ${ }^{13}{ }^{14}$ We have previously reported that NF- $\kappa \mathrm{B}$ p50 was confined to the cytoplasm distributed in the basal cells, whereas it translocates to the nuclei in the suprabasal cells of the developing conjunctiva. ${ }^{15}$ Together with the defective nuclear localisation of $\mathrm{p} 50$ and a defect in the differentiation of the conjunctiva in IkappaB kinase $\alpha(I k k \alpha)^{-/-}$conjunctiva, it was suggested that the nuclear translocation of NF- $\kappa B$ was necessary for the differentiation of the developing conjunctival epithelium.

In this study, the distribution of NF- $\mathrm{kB}$ p65 was examined in the conjunctiva of adult mouse and the human. In addition, we examined localisation of NF- $\kappa \mathrm{B}$ p65 in the conjunctival epithelium of an EKC patient infected with adenovirus as well as a mouse cornea wound model.

\section{MATERIALS AND METHOD}

\section{Animals and cornea wound model}

C57Bl6 mice (9-12 weeks old) were obtained from Hokudo Corp, Sapporo, Japan. Epithelial scraping (1 mm diameter) was performed on the right eye corneas, as previously described..$^{16}$ Briefly, an epithelial wound was created by demarcating an area on the cornea with a $1 \mathrm{~mm}$ trephine, removing the epithelium within the circle with a small scalpel, and leaving an intact basement membrane. After the injury, ofloxacin ointment was applied to the eye to avoid infection. The eyes treated in vivo after scrape wounding did not have any sign of infection when observed with a dissection microscope.

The injured corneas and conjunctiva were obtained at 6 hours after the injury and fixed immediately with $4 \%$ paraformaldehyde in $0.1 \mathrm{M}$ borate buffer $(\mathrm{pH} 9.5)$ for 2 hours and processed for paraffin section. The animal experiments conformed to the ARVO resolution on the use of animals in research.

\section{Conjunctival impression cytology}

Impression cytology specimens from two eyes of one normal human and one patient (a 25 year old woman with EKC) were retrieved from impression cytology files. Impression cytology was performed at conjunctival sites (palpebral conjunctiva) using culture membrane (Millipore Corp, Bedford, MA, USA). ${ }^{18}$ The membranes were fixed immediately with $4 \%$ paraformaldehyde in $0.1 \mathrm{M}$ borate buffer $(\mathrm{pH} 9.5)$ for 2 hours. This study was approved by the University of Hokkaido, Faculty of Medicine, and informed consent was obtained from the study subject. 


\section{Determination of adenovirus infection and typing}

The palpebral conjunctival scraps used in this study were collected from a patient whose diagnosis was EKC. In order to determine whether the patients were infected with adenovirus, we examined the infection of adenovirus using the SAS Adeno Test (SA Scientific, San Antonio, TX, USA). We also extracted DNA from these samples and performed PCR-restriction fragment length polymorphism (RFLP) analysis to determine adenovirus typing, described previously. ${ }^{19}$

\section{Immunocytochemistry}

The slides were dewaxed and rehydrated. The slides and impression cytology samples of the conjunctiva were rinsed in phosphate buffered saline (PBS) twice and incubated with normal goat serum and then with the p65 antibody (Santa Cruz, c-20; dilution, 1:200). Binding of the primary antisera was localised using FITC conjugated goat anti-rabbit IgG (dilution, 1:200; Jackson ImmunoResearch Laboratories, Inc, West Grove, PA, USA).

\section{RESULTS}

Immunocytochemistry with the anti-NF- $\kappa \mathrm{B}$ p65 antibody revealed that p65 was localised in the cytoplasm of the conjunctival epithelium of $\mathrm{C} 57 \mathrm{Bl} 6$ mouse without the treatment (fig 1A). Six hours after the scraping of the cornea, p65 protein was expressed in the nuclei of the conjunctival epithelium (fig 1B). Thus, expression of p65 protein moved from the cytoplasm to the nuclei by the treatment of scrape wounding.

By using an impression cytology technique, immunocytological analysis for p65 was adopted for the palpebral conjunctiva of the normal human (fig 2A). p65 was also localised in the cytoplasm of the conjunctival epithelium in the human normal eye (fig $2 \mathrm{~A}$ ). We also examined the distribution of p65 in the palpebral conjunctiva of the epidemic keratoconjunctivitis (EKC) patient diagnosed by the SAS Adeno Test. p65 protein was expressed in the nuclei of the conjunctival epithelial cells in the EKC patient (fig 2B).
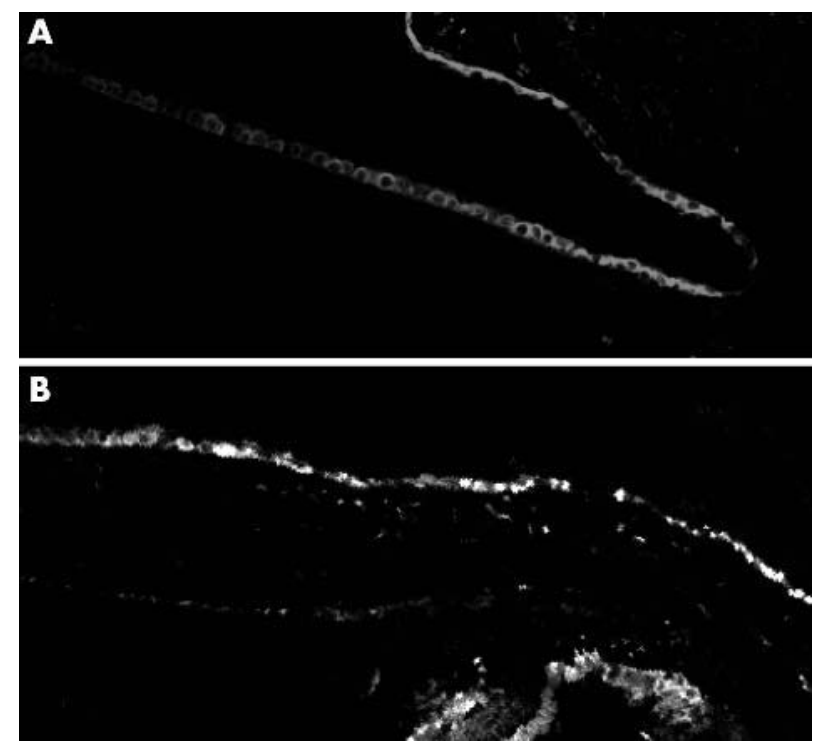

Figure 1 Immunodetection of NF- $\mathrm{kB}$ p 65 protein in the conjunctiva without treatment (A) and 6 hours after the epithelial scraping of the cornea (B) in the C57Bl6 mouse. (A) p65 protein is localised in the cytoplasm of the conjunctival epithelial cells without treatment. (B) In contrast, nuclear immunoreactivity for 065 protein is observed in the epithelium of the conjunctiva after epithelial scraping of the cornea (magnification $\times 40$ ).
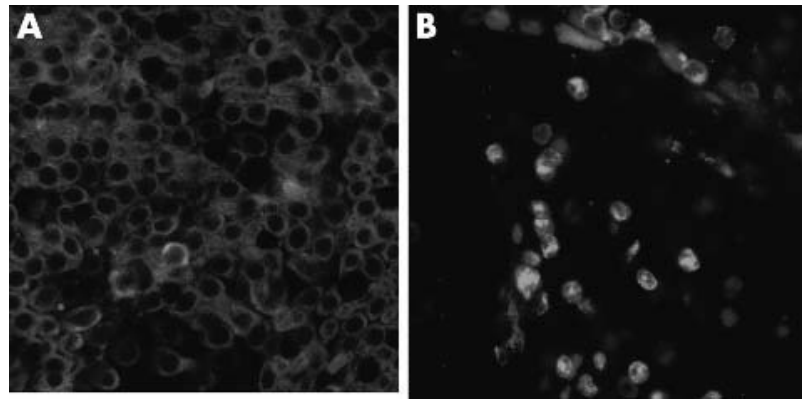

Figure 2 Immunodetection of $\mathrm{p} 65$ protein in the palpebral conjunctiva from the normal human $(A)$ and epidemic keratoconjunctivitis (EKC) patient (B). An impression cytology technique was adopted for immunocytological analysis of the conjunctival epithelium.

(A) Immunoreactivity was noted in the cytoplasm of squamous epithelial cells in a normal mucosa. (B) In contrast, nuclear immunoreactivity for p65 was noted in the squamous epithelium in an EKC patient (magnification, $\times 60$ ).

By RFLP analysis, the patient was diagnosed as adenovirus type 37 EKC (data not shown).

\section{DISCUSSION}

We have previously reported that NF- $\kappa \mathrm{B}$ p 50 was confined to the cytoplasm distributed in the basal cells whereas it translocates to the nuclei in the suprabasal cells of the developing conjunctiva. ${ }^{15}$ Together with the defective nuclear localisation of p50 and a defect in the differentiation of the conjunctiva in $I k_{k \alpha} \alpha^{-/}$conjunctiva, it was suggested that the nuclear translocation of $\mathrm{NF}-\mathrm{\kappa B}$ was necessary for the differentiation of the developing conjunctival epithelium. In this study, the distribution of NF- $\mathrm{BB}$ p65 was examined in the conjunctiva of the adult mouse and the human. In consequence, NF- $\mathrm{\kappa B}$ p 65 was distributed in the cytoplasm of the conjunctiva in each sample, suggesting that NF- $\mathrm{KB}$ p65 was not activated in the adult conjunctival epithelium.

TNF $\alpha$ and IL- $1 \beta$ both activate and are activated by NF- $\kappa B$. Thus, products of the genes that are regulated by NF- $\kappa \mathrm{B}$ also cause the activation of NF- $\kappa B .{ }^{11} \mathrm{NF}-\kappa \mathrm{B}$ acts on genes for proinflammatory cytokines including TNF- $\alpha$ and IL-1 $\beta$, chemokines (chemotactic cytokines that attract inflammatory cells to sites of inflammation), enzymes that generate mediators of inflammation, immune receptors, and adhesion molecules that play a key part in the initial recruitment of leucocytes to sites of inflammation. The activation of NF- $\kappa B$ therefore leads to a coordinated increase in the expression of many genes whose products mediate inflammatory and immune responses. This type of positive regulatory loop may amplify and perpetuate local inflammatory responses.

In contrast to the cytoplasmic distribution in the normal conjunctiva, NF- $\kappa \mathrm{B}$ p65 was translocated to the nuclei in conjunctival epithelium 6 hours after the epithelial scraping of the cornea. We also determined the nuclear expression of NF- $\kappa \mathrm{B}$ p65 in an EKC patient in this study. Together with the strong inflammation in the conjunctiva of the EKC patients as well as corneal erosion, the positive loop regarding NF- $\kappa \mathrm{B}$ may amplify and perpetuate the local inflammatory responses in the conjunctiva.

Previous reports demonstrated that corneal ulcers healed after the excision of conjunctival epithelium, ${ }^{5}{ }^{6}$ in which NF$\kappa \mathrm{B}$ p65 might be activated, as shown in this study. NF- $\kappa \mathrm{B}$ p65 is correlated with the overexpression of proinflammatory cytokines such as TNF- $\alpha$ and IL- $1 \beta$, which may represent a vigorous immune response and therefore contribute to corneal damage. ${ }^{8}$

E3 transcription unit of human adenovirus appears to be, at least in part, a cassette of genes that functions to 
counteract the host's antiviral defences. ${ }^{14}$ It was also reported that the E3 promoter had binding sites for NF- $\kappa B .{ }^{13}$ In this study, normal conjunctival epithelial cells which were not infected with adenovirus showed no nuclear expression of $N F-\kappa B$, suggesting inactivation of NF- $\kappa B$ in normal tissue. We determined that conjunctival epithelial cells revealed activation of NF- $\mathrm{\kappa B}$ in EKC. These results suggested that epithelial cells in the EKC patient escaped from host immune response via activation of NF- $\kappa \mathrm{B}$ because of the prevalence of adenovirus. The identification of mechanism that regulates the protein level of $\mathrm{NF}-\mathrm{\kappa B}$ should prove valuable in delineating the molecular pathways underlying the interaction of immune responses in corneal erosion and EKC, which involve the conjunctival epithelium.

\section{Authors' affiliations}

S Kase, K Aoki, T Harada, C Harada, K Ohgami, K Shiratori, S Ohno, K Yoshida, Departments of Ophthalmology, Hokkaido University School of Medicine, N15 W7, Kita-ku, Sapporo 060-8638, Japan

S Nishi, Departments of Biochemistry, Hokkaido University School of Medicine, N15 W7, Kita-ku, Sapporo 060-8638, Japan

Correspondence to: K Yoshida, MD, PhD, Department of Ophthalmology, Hokkaido University School of Medicine, N17 W5, Kita-ku, Sapporo, 060-8638 Japan; kyoshida@med.hokudai.ac.jp

Accepted for publication 14 July 2003

\section{REFERENCES}

1 Ford E, Warren D. Epidemiology of epidemic keratoconjunctivitis. Epidemiol Rev 1987;9:244-61.

2 Aoki K, Tagawa Y. A twenty-one year surveillance of adenoviral conjunctivitis in sapporo,Japan. Int Ophthalmol 2002;22:49-54.
3 Arnberg N, Wadell G. Adenovirus type 37 uses sialic acid as a cellular receptor on Chang C cells. J Virol 2002;76:8834-41.

4 Takeuchi S, Itoh N, Uchio E, et al. Adenovirus strains of subgenus D associated with nosocomial infection as new etiological agents of epidemic keratoconjunctivitis in Japan. J Clin Microbiol 1999;37:3392-4.

5 Parunovic A, Vuckovic S. Corneal perforation in Mooren's ulcerimmunological and clinical follow-up. Graefes Arch Clin Exp Ophthalmol 1988:226:330-1.

6 Brown SI. Therapy of Mooren's ulcer. Am J Ophthalmol 1984;98:1.

7 Sunderkotter C, Sorg C. Immunohistochemical detection of bFGF and TNFalpha in the course of inflammatory angiogenesis in the mouse cornea. Am J Pathol 1990;137:511-15.

8 Xue ML, Thakur A, Willcox M. Gene expression of pro-inflammatory cytokines and chemokines in mouse eye infected with Pseudomonas aeruginosa. Clin Exp Ophthalmol 2002;30:196-9.

9 Barnes PJ, Karin M. Nuclear factor-kappaB: a pivotal transcription factor in chronic inflammatory diseases. N Engl J Med 1997;336:1066-71.

10 Baeverle PA, Baltimore D. NF-kappa B: ten years after. Cell 1996;87:13-20.

11 Kopp EB, Ghosh S. NF-kappa B and rel proteins in innate immunity. Adv Immunol 1995;58:1-27.

12 Yoshida K, Hu Y, Karin M. IkappaB kinase alpha is essential for development of the mammalian cornea and conjunctiva. Invest Ophthalmol Vis Sci 2000:41:3665-9.

13 Williams JL, Garcia J, Harrich D, et al. Lymphoid specific gene expression of the adenovirus early region 3 promoter is mediated by NF-kappa B binding motifs. EMBO J 1990;9:4435-42.

14 Wold WS, Tollefson AE, Hermiston TW. E3 transcription unit of adenovirus. Curr Top Microbiol Immunol 1995; 199(Pt 1):237-74.

15 Yoshida K, Kim Jl, Imaki J, et al. Proliferation in the posterior region of the lens of c-maf-/- mice. Curr Eye Res 2001;23:116-19.

16 Matsuda A, Tagawa Y, Yoshida K, et al. Expression of macrophage migration inhibitory factor in rat retina and its immunohistochemical localization. J Neuroimmunol 1997;77:85-90.

17 Yoshida K, Nakayama K, Nagahama H, et al. Involvement of p27(KIP1) degradation by $\mathrm{Skp} 2$ in the regulation of proliferation in response to wounding of corneal epithelium. Invest Ophthalmol Vis Sci 2002;43:364-70.

18 Pflugfelder SC, Huang AJ, Feuer W, et al. Conjunctival cytologic features of primary Sjogren's syndrome. Ophthalmology 1990;97:985-91.

19 Saitoh-Inagawa W. Rapid diagnosis of adenoviral conjunctivitis by PCR and restriction fragment length polymorphism analysis. J Clin Microbiol 1996;34:2113-16. 